hutchinsi), have been reported from Sarqaq, on the Nugssauq Peninsula of Greenland, by Salcmonsen (1950). Alpheraky (1905) mentions a young female hybrid resulting from a cross between a White-front and Bean Goose (Anser fabalis). Another White-front $\mathrm{x}$ Snow Goose is apparently described in an early paper by Schenk (1925-26) but I was not able to examine this record. Taverner (1940) mentions that a hybrid specimen of Blue Goose $x$ White-front, taken at Fort Chipewyan, N.W.T., in 1913 , is located in the National Museum of Canada.

\section{LITERATURE CITED}

Alpheraky, Sergius. 1905. The geese of Europe and Asia. London. Rowland Ward Ltd., London.

Anonymous. 1952. Hybrids. Severn Wildfowl Trust Ann. Rept., 5:53 (see also 1950 Rept. 3:38; 1951 Rept. 4:36; 1953 Rept. 6:9; 1954 Rept. 7:53; 1957 Rept. 9:19-20).
Cooch, F. G., and J. A. Beardmore. 1959. Assortive mating and reciprocal differences in the blue-snow goose complex. Nature, 183:1833-34.

Delacour, Jean. 1954. The Waterfowl of the World. Vol. I. London, Country Life Ltd. $284 \mathrm{pp}$.

Gray, Annie P. 1958. Bird hybrids. A check-list with bibliography. Commonwealth Agricultural Bureau. Tech. Comm. No. 13. Farnham Royal.

Lonnberg, E. 1941. Further notes on some interesting goose hybrids. Arkiv for Zoologi. Vol. 33 B No. 13. (Not seen; in Salomonsen 1946.)

Salomonsen, Finn. 1946. Notes on some goose hybrids. Goteborgs Kungl Vetenskaps och Vitterhets - Samhalles Handlingar. Ser. B Band 3, No. 10: 1-14.

Salomonsen, Finn. 1950. The Birds of Greenland. Copenhagen. Ejnar Munksgaard. 608 pp.

Schenk, Jakeb. 1925-26. Feher vadludak magvarorszager on - Weisse wildganse in Ungarn. Aquila, 32-33: 139-146.

Sibley, C. L. 1938. Hybrids of and with North American Anatidae. Proc. Intern. Ornith. Congr. 9: 327-335. Rouen.

Taverner, P. A. 1940. The nesting of Ross's Goose Chen rossi. The Canad. Field-Nat., 54 (9): $127-130$.

\title{
Fourth Annual May Day Bird Count
}

\section{Regina Natural History Society, May 16, 1964}

The Regina May bird census on May 16, 1964, recorded 141 species of birds in an area approximating the circle of 15-mile radius described for the Birds of Regino (1961). Vic Wilshire was in charge of organizing the parties in the six zones. Of special interest was the observation for the first time in the Regina area, of a Brewer's Sparrow (see article by R. W. Nero, P. 99).

SPECIES LIST: Red-necked Grebe 4; Horned Grebe, 39; Eared Grebe, 117; Western Grebe, 19; Pied-billed Grebe, 10; Double-crested Cormorant, 3; Great Blue Heron, 2; Blackcrowned Night Heron, 18; Mute Swan, 5; Whistling Swan, 5; Trumpeter Swan, 1; Canada Goose, 245 (including 90 young); Mallard, 314; Gadwall, 77; Pintail, 79; Green-winged Teal, 18; Blue-winged Teal, 320; American Widgeon, 182; Shoveler, 94; Redhead, 40; Ring-necked Duck, 1; Canvasback, 83; Lesser Scaup, 100; Common Goldeneye, 1; Bufflehead, 1; Ruddy Duck, 79; Red-tailed Hawk, 3; Swainson's Hawk, 5; Marsh Hawk, 4; Sharptailed Grouse, 13; Ring-necked Pheasant, 4; Gray Partridge, 42; Sora, 16; American Coot, 189; Semipalmated Plover, 1; Killdeer, 89: American Golden Plover, 474; Black-bellied Plover, 9; Ruddy Turnstone, 1; Common Snipe, 2; Upland Plover, 8; Spotted Sandpiper, 14; Solitary Sandpiper 6; Willet, 48; Lesser Yellowlegs, 17; Pectoral Sandpiper, 274; Whiterumped Sandpiper, 4; Baird's Sandpiper, 11; Least Sandpiper, 73; Long-billed Dowitcher, 32; Stilt Sandpiper, 11; Semipalmated Sandpiper, 36; Marbled Godwit, 36; Sanderling, 21 ; Ámerican Avocet, 15; Wilson's Phalarope, 170; Northern Phalarope, 35; Ring-billed Gull' 47; Franklin's Gull, 155; Common Tern, $40+$; Black Tern, 292; Rock Dove, 54; Mourning Dove, 60; Great Horned Owl, 3; Burrowing
Owl, 7: Short-eared Owl, 1: Common Nighthawk, 3; Belted Kingfisher, 1; Yellow-shatted Flicker, 9 (plus 3 hybrids); Ked-shatted Flicker, 1; Downy Woodpecker 1; Eastern Kingbird, 37; Western Kingbird, 73; Eastern Phoebe, 1: Say's Phoebe, 3; Traill's Flycatcher, 11; Least Flycatcher, 47 (with 24 Empidonax not identified): Western Wood Pewee, 3; Olivesided Flycatcher, 1; Horned Lark, $425 \pm$; Tree Swallow, 51 $\$$; 'Bank Swallow, 24; Barn' Swallow, 86; Cliff' Swallow, 1; Purple Martin, 26; Black-billed Magpie 61; Common Crow, 126; Red-breasted Nuthatch, 1; House. Wren, 8; Brown Thrasher, 34; Robin, 81; Swainson's Thrush, 56; Grey-cheeked Thrush, 13; Veery, 1; Ruby-crowned Kinglet, 3; Water Pipit, 1; Cedar Waxwing, 1; Loggerhead Shrike, 20; Starling, 18; Solitary Vireo, 1; Philadelphia Vireo 1; Warbling Vireo, 2; Tennessee Warbler, 10; Orange-crowned Warbler, 23; Yellow Warbler, 112; Magnolia Warbler, 1; Myrtle Warbler, 53; Blackpoll Warbler, 6; Palm Warbler, 2; Northern Waterthrush, 5; Mourning Wárbler, 1; Yellowthroat, 8; American Redstart, 9; House Sparrow 395t; Western Meadowlark, 192; Yellow-headed Blackbird 220; Red-winged Blackbird, 971; Baltimore Oriole, 50; Brewer's Blackbird, 172; Common Grackle, 92 + ; Brown-headed Cowbird, 193; Rose-breasted Grosbeak, 4; American Goldfinch, 2; Rufous-sided Towhee, 14; Lark Bunting, 41: Savannah Sparrow, 73; Baird's Sparrow, 3; Vesper Sparrow, 69; Lark Sparrow 1; Chipping Sparrow, 646; Clay-colored Sparrow, 837; Brewer's Sparrow, 1; Harris' Sparrow, 41; White-crowned Sparrow, 61; White-throated Sparrow, 18; Lincoln's Sparrow, 14; Swamp Sparrow, 3; Song Sparrow, 29; McCown's Longspur, 69; Lapland Longspur, 3246; Chestnut-collared Longspur, 77. - Dorothy Wode, compiler. 